\title{
A cross-section observational study on the seroprevalence of antibodies to COVID-19 in patients receiving opiate agonist treatment
}

\author{
Fiona Fenton $^{1}\left[\right.$ D $\cdot$ Siobhan Stokes ${ }^{1} \cdot$ Marie Eagleton $^{1}$ \\ Received: 23 February 2021 / Accepted: 26 April 2021 / Published online: 9 July 2021 \\ (c) Royal Academy of Medicine in Ireland 2021, corrected publication 2021
}

\begin{abstract}
Introduction The HSE National Drug Treatment Centre is an inner city drug treatment centre in Dublin which provides opiate agonist treatment (OAT) to approximately 565 patients, many of whom have complex care needs.

Objective This study was conducted to determine seropositivity to the COVID-19 virus in patients attending NDTC, and to establish if patients tested had any clinical symptoms of this disease since March 2020.

Method All patients attending for OAT were invited to participate and 103/565 patients agreed. The patients were tested for the presence of serum antibodies to COVID-19 in a single sample collected over a 4-month period (July-October 2020). A questionnaire was administered at the same time as sample taking.

Results Results showed that the majority of patient samples $(100 ; 97 \%)$ tested were negative for the presence of antibodies to COVID-19. There were only two confirmed positive results (1.9\%) and one equivocal result (1\%). None of the approximately 565 attendees at the HSE NDTC presented with serious illness indicative of COVID-19 throughout the three waves of the pandemic, nor were any deaths due to COVID-19 reported.

Conclusion These findings indicate (a) possible low level of exposure to COVID-19 among this patient cohort or (b) that those patients who have been exposed have not developed or maintained detectable antibody levels, nor developed symptoms of the disease. Public health measures could explain the low level of COVID-19 in this cohort. The findings are also consistent with the possibility of a protective effect of OAT medications on development of the disease.
\end{abstract}

Keywords Buprenorphine $\cdot$ COVID-19 $\cdot$ Methadone $\cdot$ Opiate substitution treatment $\cdot$ OST $\cdot$ Protective $\cdot$ SARS-CoV-2 . Seroprevalence

\section{Introduction}

\section{Background/rationale}

With the COVID-19 pandemic, it was greatly feared that the virus would sweep through vulnerable populations. People with a drug dependency were widely considered to be at particular risk for infection with this virus [1-4]. Despite expectations to the contrary [4-6], there have been very few reports internationally of COVID-19 in people who use drugs (PWUD) treated with OAT [7-9]. The European Monitoring Centre for Drugs and Drug Addiction [8] provided a snapshot of the current situation re COVID-19 in

Fiona Fenton

ffenton@dtcb.ie

1 HSE National Drug Treatment Centre, 30-31 Pearse St, Dublin 2, D02 NY 26, Ireland addiction populations. Despite an estimated 654,000 opioid users in Europe receiving OAT, most health professionals surveyed did not report cases: seven reported five or fewer cases, two reported ten or more cases and one Belgian doctor reported only two deaths among OAT patients. A Dublin-based case study in the same EMCDDA report on a cohort of homeless drug users showed an unexpectedly low incidence of COVID-19 and no deaths. No evidence is presented of increased morbidity or mortality in this population attributable to COVID-19. The main risk identified by these authors for patients with OUD during the pandemic relates to increased risk of drug overdose due to changes in service delivery and drug market changes. It has thus been suggested that it would be of interest to test for COVID-19 antibodies in this population $[9,10]$.

As a response during the first wave, the combined efforts of local authority, housing first tenancies and NGOs meant a reduction in people accessing everyday homeless accommodation. The health authority (HSE) produced guidelines to 
prevent viral spread in vulnerable groups with an emphasis on homeless and group settings.

We have postulated that OAT has a protective effect on the clinical manifestations of COVID-19 and called for further research on this [7]. Given the widespread concern about COVID-19 amongst PWUD, establishing the rate of exposure to COVID-19 in this population is important from a public health perspective, and in understanding how to optimally protect vulnerable groups.

\section{Aims and objectives}

The aim of the study was to determine seropositivity to the COVID-19 virus in patients attending NDTC and to compare their level of seropositivity with data published about the general Irish population. Additionally, the study aimed to establish if patients displayed any clinical symptoms of COVID-19 (Table 1).

\section{Methods}

\section{Study design}

Patients on OAT attending an inner city Dublin drug treatment centre were invited to participate in the study over a period of 4 months (July October 2020). Informed consent was obtained from each participant who agreed to take part in the study. A patient information leaflet on the study and COVID-19 was given to each participant. A single blood sample was collected and tested for antibodies to COVID-19. A questionnaire on the presence of COVID-19 symptoms, current medication, underlying conditions, smoking history and contact with a known COVID-19 case was administered at the same time. Serology testing was carried out in the National Virus Reference Laboratory (NVRL) at University College Dublin. Ethics approval for the study was obtained from the Office for National Research Ethics Committee (NREC-COV-065) in July 2020. Data collection was done by the principal investigator. Data was pseudonymised, and each dataset was identified by number.

\section{Setting}

The HSE National Drug Treatment Centre (NDTC) provides opiate agonist treatment (OAT), principally methadone or buprenorphine with naloxone to approximately 565 patients, many of whom have underlying viral illnesses such as hepatitis C, HIV and chronic respiratory conditions. The study commenced in the HSE National Drug Treatment Centre at the end of July 2020 and ran for 3 months. There are an estimated 11,000 opioid-dependent patients registered in Ireland and approx 565 of these attend the NDTC. Over the timescale of the study, there were
Table 1 Describes the incidence of COVID type symptoms, smoking status, details of OAT, underlying conditions and prescribed medications in a cohort of patients tested for antibodies to COVID-19. The numbers and percentage of total are given in brackets

Clinical data $N=103$

(n)

(\%)

Symptoms since March 2020

$\begin{array}{ll}\text { Fever } & 16(15) \\ \text { Cough } & 29(19) \\ \text { Dyspnoea } & 26(25) \\ \text { Fatigue } & 35(34) \\ \text { Myalgia } & 20(19) \\ \text { Anosmia/Hypogeusia } & 12(11.5) \\ \text { ore throat } & 7(6.7) \\ \text { Headache } & 18(17) \\ \text { Rhinorrhoea } & 22(21) \\ \text { Sneezing } & 9(8.6) \\ \text { Smoker } & 97(94) \\ \text { Non-smoker } & 6(6)\end{array}$

OAT

Suboxone (total no.) $11(11)$

Methadone (total no.) 92 (89)

Suboxone $12 \mathrm{mg}$ or over (MTD*) $3(3)$

Methadone $60 \mathrm{ml}$ or over (MTD*) 71 (69)

MTD* $=$ minimum therapeutic dose

Underlying conditions

Hepatitis C 12 (11.6)

HIV 7 (7)

Respiratory 18 (17.5)

Cardiovascular $3(2.9)$

Thyroid $1(0.9)$

Diabetes $3(0.97)$

Prescribed medication

Antipsychotic 23 (22)

Hypnotic/sedative 43 (42)

SSRI $41(40)$

Antiepileptic $12(11.6)$

Antivirals $\quad 7(6.7)$

Respiratory $3(2.9)$

GI 15 (14.5)

Vitamins 41 (40)

Cardiovascular $1(0.9)$

NSAIDS 2 (1.9)

Thyroid $1(0.97)$

two waves of COVID-19 in Ireland. Public health measures such as social distancing, mask wearing and enhanced cleaning started on March 16th in the NDTC. Full lockdown began on March 27th and was extended until May 5 th (first wave). The second wave which has been deemed by HPSC to have commenced on 2 nd August initiated a further lockdown on October 22nd for 6 weeks. 


\section{Serology testing}

A serum sample from each patient was sent to the National Virus Reference Laboratory (NVRL) for testing. Each sample was tested for antibodies to SARS-CoV-2 using two commercial assays: the Abbott Architect SARS CoV-2 IgG Assay which detects antibodies (IgG) to the SARS CoV-2 nucleocapsid protein and the FORTRESS Diagnostics SARS-CoV-2 ELISA, which detects total antibody response to the receptor-binding domain of SARS-CoV-2 spike protein. If the results were discordant between the two assays, the sample was tested on a third assay, the Liaison assay, which detects antibodies to spike 1 and 2 protein. It there was concordance between the FORTRESS and Liaison assays that result was accepted, if there was discordance, the result was considered equivocal.

The Abbott Architect SARS-CoV-2 IgG Assay used to detect antibodies (IgG) to SARS-CoV-2 has a sensitivity and specificity of $93 \%$ and $100 \%$, respectively.

The Fortress Diagnostics COVID-19 Total Antibody Assay has a sensitivity range of 94 to $95.4 \%$ and a specificity of $100 \%$.

The DiaSorin LIAISON SARS-CoV-2 S1/S2 IgG assay has, at greater than 15 days post COVID-19 diagnosis, a sensitivity of $97.9 \%$ and a clinical specificity of $98.6 \%$.

\section{Participants}

All patients attending the HSE National Drug Treatment Centre clinic are on OAT with their scheduled frequency of attendance varying depending on their individual care plan. Posters about the study were displayed in the clinic. Each patient was asked to participate once only and was under no duress to participate. If a patient refused to participate, this was recorded and highlighted in their care plan, so that they would not be asked again. All participants were assessed for their individual medical decision making capacity using a standardised assessment and voluntarily gave informed consent prior to being enrolled. When a patient consented, a questionnaire was administered followed by the drawing of one $5 \mathrm{ml}$ serum clotted blood sample.

Exclusion criteria were lack of willingness to participate, or the lack of ability to provide informed consent.

\section{Variables}

Patients were asked if they had experienced any of the common symptoms of COVID-19 since early March 2020. These included fever or chills, cough, shortness of breath, fatigue, aches and pains, new loss of taste and/or smell, sore throat, headache, runny or stuffy nose, nausea or vomiting, diarrhoea or sneezing. Also recorded were respondent's age, sex, smoking history, prescribed medication, any underlying conditions and whether they had been in recent contact with anyone who had COVID-19.

\section{Bias}

To avoid selection bias, patients were recruited for the study consecutively by either a nurse or a doctor. Participants' willingness to participate in the study may have been a factor in that more co-operative subjects volunteered.

\section{Causal inference}

We describe a study of a unique group on OAT who are typically a high risk and vulnerable population for all infections. Our sampling strategy was to avoid selection bias.

\section{Results}

\section{Study size/participants}

One hundred and three (103) patients on OAT attending the clinic located in inner city Dublin agreed to participate and were tested for antibodies to SARS-CoV-2 over a period of 4 months (July-October 2020). Many of these patients have complex care needs including underlying viral illnesses such as hepatitis $\mathrm{C}$ and HIV, as well as chronic respiratory conditions. This sample size represents $\sim 18 \%$ of all attendees at our centre and $\sim 0.93 \%$ of all patients on OAT in Ireland. Due to low numbers of patients in the study, we did not apply statistical methods.

Thirty-seven females (36.5\%) and sixty-six males (63.5\%) participated. The age range was $25-64$ with a median age of 39.5 years. The median age for females was 35.5 years, and the median age for males was 38 years. The following symptoms were reported retrospectively on completing the questionnaire but had not been presented previously as possible signs of COVID-19 infection: fatigue $34 \%$, rhinorrhoea $21 \%$, cough and myalgia $19 \%$, and headache $17 \%$. In addition the following coexisting conditions, treatments were reported in the patient group: chronic respiratory disease asthma or COPD, $17.5 \%$; hepatitis $\mathrm{C}$ infection, $11.5 \%$; and treatment with antiviral drugs, $7 \%$. Five patients $(5 \%)$ reported being in contact with confirmed cases of COVID-19.

Eleven patients (11\%) were treated with buprenorphine/ naloxone, and ninety-two (89\%) were treated with methadone. In total, $72 \%$ were on therapeutic doses of OST. The remaining $28 \%$ were on subtherapeutic doses.

Forty-one patients (40\%) were given regular multivitamins which is indicative of chronic poor nutritional status. Twenty-three patients (22\%) were on antipsychotic drugs either for dual diagnosis, ongoing symptoms of druginduced psychotic symptoms or as anxiolytics. 
Ninety-seven of the patients (94\%) in this group were smokers.

\section{Serology testing}

The antibody test results of the 103 patients tested for antibodies to COVID-19 are summarised in Table 2. Ninetynine patients tested were negative in both the Abbott and Fortress assays and were reported as negative for antibodies to COVID-19. One patient was positive in the Abbott assay but negative by both the FORTRESS and Liaison assays and was reported as negative for antibodies to COVID-19, giving an overall negative rate for the population tested of 97\%. One patient tested was negative by the Abbott assay but was positive in both the FORTRESS and Liaison Assays and was reported as positive for antibodies to COVID-19. A second patient was equivocal on the Abbott assay but positive on the Fortress assay and was reported as positive for antibodies to COVID-19, giving an overall positivity rate in the population tested of $1.9 \%$. One patient was negative by both the Abbott and Liaison assays but was positive by the FORTRESS assay and was reported as indeterminate or equivocal $(0.1 \%)$.

In summary, antibodies to COVID-19 were not detected in the majority of patients tested (97\%); conversely, the confirmed seropositivity in the population was less than $2 \%$.

\section{Individual cases}

One confirmed positive serology result in our study was a 29-year-old man on $25 \mathrm{mg}$ (low dose) of methadone who was released from prison in April 2020 and returned to custody after three days. He immediately developed symptoms including fever, sore throat and diarrhoea which he thought at the time was COVID-19. These symptoms lasted overnight and were resolved the following morning. He had no underlying conditions and reported no known contact with COVID-19. He was not tested for COVID-19 at the time.

One equivocal serology result was determined in a 32-year-old man who said he may have been in contact with a COVID-19 case but who never developed symptoms. He was on methadone $80 \mathrm{mg}$ and had no underlying conditions. He said his possible contact was unreliable.

Table 2 Describes the COVID-19 antibody test results for all participating patients. SARS CoV-2 serology result in a cohort of 103 patients treated with opiate agonist therapy

\begin{tabular}{lc}
\hline SARS-CoV-2 antibody test status & $N(\%)$ total 103 \\
\hline Antibody-positive & $2(1.9)$ \\
Antibody-negative & $100(97)$ \\
Equivocal & $1(0.1)$ \\
\hline
\end{tabular}

One 41-year-old female patient had a positive IgG serology result in October 2020. She had a positive PCR test done routinely in April 2020 whilst in hospital for treatment of a groin abscess and secondary pneumonia related to intravenous drug use. She has a history of recurrent blood clots and is on prophylactic anticoagulants. She was on $60 \mathrm{mg}$ methadone and has been treated successfully for hepatitis $\mathrm{C}$ in the past. She was considered a weak phenotype of COVID-19 during her stay in hospital. Hypercoagulopathy is recognised as a third phase in COVID-19 disease [5].

Five patients answered that they had contact with a known case of COVID-19, but they did not develop the disease and tested negative on serology. One of those had asthma and one had hepatitis $\mathrm{C}$. The remaining three patients who reported contact with a known case had no underlying coexisting conditions. Both positive cases and the one equivocal result had no confirmed contact with a COVID-19 case nor had they clinically significant COVID-19 disease.

\section{Discussion}

The majority of patient samples (100 of 103) tested were negative for the presence of antibodies to COVID-19. Importantly, none of the attendees at HSE- NDTC has experienced serious illness from COVID-19, and there have been no deaths to date throughout three waves of the pandemic.

The second wave of COVID-19 in Ireland has been deemed to have commenced on 2nd August 2020, but the lockdown was not initiated until October 22nd 2020. Restrictions had been eased in Ireland in the period of the study, and the patients were attending the clinic. The patients were therefore broadly at a similar risk of exposure to COVID-19 as the rest of the Dublin general population, but at a theoretically higher risk due to routine sharing of drug paraphernalia, cigarettes, and masks and personal challenges in maintaining social distancing.

The two antibody-positive patients were on low dose OAT and experienced symptoms that were consistent with COVID-19 infection, but in both cases, the symptoms resolved quickly, and treatment was not required. The third patient whose results were equivocal did not experience symptoms. Of interest given the high contagiousness of the COVID-19 virus is the observation that five patients; two of whom had significant comorbidities and reported known contact with COVID-19 but did not develop the disease.

The low seropositivity rate and low morbidity in those who tested positive are interesting given the co-morbidities and increased risk factors for COVID-19 present in this cohort. Research has shown that those with mental disorders (not on OAT) [11] are most vulnerable to COVID-19 infection. Of note is that $22 \%$ of the group were prescribed antipsychotic medications, $42 \%$ were prescribed hypnotics/ sedatives and $40 \%$ were prescribed SSRIs. 
The group has other increased risk factors and comorbidities: the majority of patients $(94 \%)$ in the group smoked tobacco which is one of the most important risk factors for COVID-19 transmission and outcome [12]. Nicotine and tobacco smoke can upregulate ACE2 receptors in different organs of the body, and ACE2 has been established as the receptor by which the virus enters the host cells [13]. Additionally, $17 \%$ of the population has underlying respiratory conditions, and $11 \%$ had hepatitis $\mathrm{C}$ disease. Increased age is recognised as the most significant risk factor for mortality and hospitalisation with COVID-19. Whilst the median age of study participants is relatively low at 39.5 years (range 25-64), it matches the median age of confirmed positive cases in the general population in Ireland which is 39 years. People in the age range 25-64 also represented 35,649 (59\%) of the 60,287 confirmed COVID-19 cases notified up to the end of October 2020 and 1836 (42\%) of the 4348 people hospitalised with COVID-19 [14].

Comparing results of the Scopi study [15], which demonstrated a prevalence of COVID-19 in the general population in Ireland of 1.7\% and 3.1\% in Dublin, with the results of this study, is of interest. The Scopi study was carried out over the period June-July 2020 before our study commenced. By the end of July, there had been 12,505 confirmed cases of COVID-19 in Dublin; the curve of the first wave of the pandemic in Ireland had essentially been flattened with less than 50 new cases per day being reported [16]. By contrast, the present study largely coincided with the second wave of the pandemic in Ireland which peaked in mid-October when the number of new cases per day ranged from 800 to 1300 [14] therefore requiring the implementation of further lockdown measures. Thus, by the end of October 2020, the second wave of COVID-19 infection in Ireland had progressed to the extent that numbers of confirmed cases in the Dublin population had almost doubled to 23,305 [14]. It is therefore likely that the SARS COV-2 seroprevalence in the general population in Dublin was higher than $3.1 \%$ by the end of October when our study reached completion. We found the seroprevalence in our group to be lower at $1.9 \%$.

One possibility for the observation of low/no COVID-19 disease is that none of the group has been in contact with a COVID-19 infected person and therefore has not become infected. Protective measures such as providing hotel accommodation for homeless people were taken, and arguably public health measures could explain the low level of COVID19 in this cohort. A high level of cocooning of this cohort occurred in the first wave including provision of takeaway medication; after the first wave had passed and over the period of our study, patients had returned to regular attendance at the clinic, albeit with ongoing public health measures. The expectation that COVID-19 would spread rapidly through the OAT patient population did not materialise here or elsewhere. Any reported outbreaks were in addiction clinics (two in Dublin, personal knowledge, one in Germany occurred in staff, not in patients attending) [17].

We believe that the findings are representative of a correspondingly low incidence of COVID-19 in the rest of the OAT population in Ireland and elsewhere. Whilst it is possible that targeted protective measures in combination with public health measures have protected this group resulting in a low seroprevalence and disease level, it may not fully explain the low level of seroprevalence to COVID-19. Many of patients engage in behaviours that may increase the risk of exposure to COVID-19: sharing masks, drug paraphernalia, poor observation of social distancing etc. The findings are also consistent with the possibility of a protective effect of OAT medications on development of the disease [7]. Whatever the reason for low seroprevalence and COVID-19 disease, the expectation that COVID-19 would spread rapidly through the OAT patient population has not materialised here or elsewhere. We have found no significant reports of outbreaks of the disease in patients on OAT.

OAT is a key component in the treatment of opioid dependence and plays an important role in rehabilitation and recovery. Importantly, engaging patients in OAT provides stability and support that protect people. We would encourage healthcare services in all countries to engage as many opioid-dependent individuals as possible on OAT. Engaging with services and continued social support for this group of patients throughout this pandemic is an important public health strategy in maintenance of their health. Provision of OAT for those not already on this treatment may help keep levels of COVID-19 disease low in this population.

\section{Limitations}

This study ran from late July to the end of October. We acknowledge the small sample size and consequent lack of statistical analysis. Patients were asked consecutively to participate to avoid selection bias. Those who agreed may be more health-motivated individuals.

\section{Generalisability}

Despite the anticipated vulnerability to COVID-19 infection in drug-using patients, there is a lack of international reports of COVID-19 in these groups [9]. The reasons for this are unclear.

A search on PubMed with the following terms (COVID19) AND (methadone) revealed forty papers, of these thirtyseven related to measures taken to ensure continuity of treatment, one regarding fatalities due to overdose and two regarding COVID-19 outbreaks in PWUD. 


\section{Conclusion}

Our study demonstrated a very low seroprevalence to COVID-19 antibodies in a cohort of patients treated with OAT, a group considered to be very vulnerable to such infection if exposed to the virus. None of the attendees at HSE-NDTC has experienced serious illness from COVID19 , and there have been no deaths due to COVID-19 to date throughout three waves of the pandemic. The finding of $1.9 \%$ seropositivity to COVID-19 in this population is lower than anticipated and consistent with the low level of COVID19 reported in this patient cohort in Ireland and elsewhere. Further research into COVID-19 in the OAT population is warranted.

Acknowledgements The following nurses in NDTC who consented patients and took blood samples: Sonia Bravo, Jane Bridgeman, Bernie Carthy, Jane Gahan. Helen Hannon, Mary Maloney, Aoife Muldoon, Sorcha O'Beirne, Marie O'Sullivan, Brid Mac Dermott, Clara Rubio, Aileen Singleton, Juliana Soria, Lorealle Zayco. Also the following doctors: Margaret Bourke, Darren Daly, Barbara Duffy, Cristina Herteu, Laura Mangan, Deepak Prashant, Siobhan Sheehy, Kiran Santlal, Megan McNicholas.

Advice and support from Dr Kevin Ducray, Senior Psychologist, NDTC.

This study was done in collaboration with Dr Cillian de Gascun and Paul Holder, UCD NVRL.Thanks are due to the laboratory staff at UCD NVRL for conducting serology testing and laboratory staff at the NDTC for facilitating sample management.

\section{References}

1. Dubey M, Ghosh R, Chatterjee S et al (2020) COVID-19 and addiction

2. Schimmel J, Manini A (2020) Opioid use disorder and COVID-19: biological plausibility for worsened outcomes. Subst Use Misuse 55(11):1900-1901

3. Volkow N (2020) Collision of the COVID-19 and addiction epidemics. Ann Intern Med 173(1):61-62

4. Wang Q, Kaelber D, Xu R, Volkow N (2020) COVID-19 risk and outcomes in patients with substance use disorders: analyses from electronic health records in the United States. Mol Psychiatry. https://doi.org/10.1038/s41380-020-00880-7
5. Ataei M, Shirazi F, Lamarine R et al (2020) A double-edged sword of using opioids and COVID-19: a toxicological view

6. Lambert D (2020) Opioids and the COVID-19 pandemic: does chronic opioid use or misuse increase clinical vulnerability? Br J Anaesth 125(4):e382-e383

7. Eagleton M, Stokes S, Fenton F, Keenan E (2020) Does opioid substitution treatment have a protective effect on the clinical manifestations of COVID-19? Comment on Br J Anaesth. https://doi. org/10.1016/j.bja.2020.11.027

8. EMCDDA (2020) [Internet]. Emcdda.europa.eu. [cited 12 November 2020]. Available from: https://www.emcdda.europa.eu/ system/files/publications/13073/EMCDDA-Trendspotter-Covid19_Wave-1-2.pdf

9. Welle-Strand G, Skurtveit S, Clausen T et al (2020) COVID-19 survey among people who use drugs in three cities in Norway. Drug Alcohol Depend 217:108302

10. Vallecillo G, Perelló R, Güerri R et al (2020) Clinical impact of COVID-19 on people with substance use disorders. J Public Health

11. Yao H, Chen J, Xu Y (2020) Patients with mental health disorders in the COVID-19 epidemic. Lancet Psychiatry 7(4):e21

12. Sifat A, Nozohouri S, Villalba $\mathrm{H}$ et al (2020) The role of smoking and nicotine in the transmission and pathogenesis of COVID-19. J Pharmacol Exp Ther 375(3):498-509

13. Bourgonje AR, Abdulle AE, Timens W et al (2019) Angiotensinconverting enzyme-2 (ACE2), SARS-CoV-2 and pathophysiology of coronavirus disease (COVID-19). Journal of Pathology, Invited Review. https://doi.org/10.1002/path.5471

14. https://www.hpsc.ie/a-z/respiratory/coronavirus/novelcoronavirus/casesinireland/epidemiologyofcovid-19inireland/october2020/ COVID-19_Daily_epidemiology_report_(NPHET)_20201030\% 20-\%20website.pdf. Accessed 16 April 2021

15. Study to investigate COVID-19 infection in people living in Ireland (SCOPI) (2020) Journal of Pathology, Invited [Internet]. Hpsc.ie. [cited 21 November 2020]. Available from: https:// www.hpsc.ie/a-z/respiratory/coronavirus/novelcoronavirus/scopi/ SCOPI\%20report\%20preliminary\%20results\%20final\%20version. pdf

16. https://www.hpsc.ie/a-z/respiratory/coronavirus/novelcoronavirus/casesinireland/epidemiologyofcovid-19inireland/july2020/ COVID-19_Daily_epidemiology_report_(NPHET)_31072020\% 20-\%20Website.pdf. Accessed 16 April 2021

17. IOTOD annual-conference 24-25 September 2020 Dr Stephan Walcher Director, Concept Centre for Addiction Medicine, Munich, Germany

Publisher's Note Springer Nature remains neutral with regard to jurisdictional claims in published maps and institutional affiliations. 